\title{
Influência da precipitação e do uso do solo nas vazões da Sub-Região Hidrográfica Itacaiúnas (SRHI)
}

\author{
Influence of precipitation and land use on flows in the Hydrographic Sub-Region Itacaiúnas \\ (SRHI) \\ Influencia de la precipitación y uso de la tierra en los flujos de la SubRegión Hidrográfica \\ Itacaiúnas (SRHI)
}

\author{
Kelvis Nunes da Silva \\ ORCID: https://orcid.org/0000-0003-1085-5494 \\ Universidade Federal do Pará, Brasil \\ E-mail: kelvisnsilva@gmail.com \\ Eduarda Costa Ferreira \\ ORCID: https://orcid.org/0000-0001-9094-1151 \\ Universidade do Estado do Pará, Brasil \\ E-mail: eduardacostaferreira5@gmail.com \\ Juliana Cardoso Ferreira \\ ORCID: https://orcid.org/0000-0002-5176-7135 \\ Universidade do Estado do Pará, Brasil \\ E-mail: juliana.cferreira@aluno.uepa.br \\ Vanessa Silva Oliveira \\ ORCID: https://orcid.org/0000-0002-5403-1932 \\ Universidade Federal do Pará, Brasil \\ E-mail: voliveiraambiental@gmail.com \\ Mateus dos Santos Coelho \\ ORCID: https://orcid.org/0000-0002-3411-3260 \\ Universidade do Estado do Pará, Brasil \\ E-mail: Mateusc_27@hotmail.com \\ Virginia Bezerra Rodrigues \\ ORCID: https://orcid.org/0000-0001-5315-6517 \\ Universidade Única - Instituto Paragominas, Brasil \\ E-mail: virginiarodrigues89@gmail.com \\ Glauber Epifanio Loureiro \\ ORCID: https://orcid.org/0000-0002-0801-5296 \\ Universidade do Estado do Pará, Brasil \\ E-mail: epfanio@uepa.br
}

\begin{abstract}
Resumo
No decorrer dos últimos anos as bacias hidrográficas sofreram alterações substanciais em seu comportamento hidrológico assim como nas condições de uso e ocupação do solo devido atividades econômicas. Nesse sentido, o objetivo do presente estudo é analisar e caracterizar os efeitos da precipitação e do uso e ocupação do solo na vazão da Sub-Região Hidrográfica Itacaiúnas (SRHI) entre os períodos de 1988 a 2017. Para isso, informações referentes às séries temporais de vazão e precipitação no período de 30 anos foram obtidas das estações que se encontram na região que são disponibilizadas no site da Agencia Nacional de Águas (ANA). Além disso, também foram obtidos dados de uso e ocupação do solo do projeto MapBiomas com o mesmo período de 30 anos. Posteriormente realizaram-se correlações entre precipitações e vazões assim como para o uso e ocupação do solo e vazões. Com isso, observou-se que as vazões apresentaram estreita relação com a precipitação, no entanto, o uso do solo não apresentou correlação expressiva com a dinâmica da vazão na região.
\end{abstract}

Palavras-chave: Variáveis climáticas; Mapeamento; Meio Ambiente.

\section{Abstract}

Over the last few years, the hydrographic basins have undergone substantial changes in their hydrological behavior as well as in the conditions of use and occupation of the soil due to economic activities. In this sense, the objective of this study is to analyze and characterize the effects of precipitation and land use and occupation on the flow of the Itacaiúnas Hydrographic Sub-Region (SRHI) between the periods 1988 to 2017. For this, information regarding the series flow and precipitation storms over a 30-year period were obtained from stations located in the region, which are 
available on the website of the National Water Agency (ANA). In addition, land use and occupation data from the MapBiomas project were also obtained for the same period of 30 years. Subsequently, correlations were made between rainfall and flow, as well as for land use and occupation and flow. With that, it was observed that the outflows had a close relationship with the precipitation however, the use of the land did not present expressive correlation with the dynamics of the flow in the region.

Keywords: Climatic variables; Mapping; Environment.

\section{Resumen}

En los últimos años, las cuencas hidrográficas han experimentado cambios sustanciales en su comportamiento hidrológico, así como en las condiciones de uso y ocupación del suelo debido a las actividades económicas. En este sentido, el objetivo de este estudio es analizar y caracterizar los efectos de la precipitación y el uso y ocupación del suelo sobre el caudal de la Subregión Hidrográfica Itacaiúnas (SRHI) entre los períodos 1988 a 2017. Para ello, información relativa a la serie Las tormentas de flujo y precipitación durante un período de 30 años se obtuvieron de estaciones ubicadas en la región, las cuales están disponibles en el sitio web de la Agencia Nacional del Agua (ANA). Además, también se obtuvieron datos de uso y ocupación del suelo del proyecto MapBiomas para el mismo período de 30 años. Posteriormente, se realizaron correlaciones entre lluvia y caudal, así como para uso y ocupación del suelo y caudal. Con eso, se observó que los desagües tuvieron una estrecha relación con la precipitación, sin embargo, el uso del terreno no presentó correlación expresiva con la dinámica del flujo en la región.

Palabras clave: Variables climáticas; Cartografía; Medio Ambiente.

\section{Introdução}

As alterações na cobertura do solo da região Amazônica acontecem de maneira ativa ao longo dos anos, devido a substituição de áreas de floresta por pastagem, além da exploração dos recursos naturais que são fatores concomitantes para a redução do recurso (Delazeri, 2016). Segundo Vieira et al., (2014) a floresta amazônica possui a maior extensão de florestas tropicais e biodiversidade do mundo.

No decorrer dos últimos anos, as bacias hidrográficas sofreram alterações substanciais em seu comportamento hidrológico (Adami et al., 2015). Tais mudanças em sua maioria, ocorrem devido às mudanças climáticas, alterações nas características da precipitação, bem como ações antrópicas diretas decorrentes de por atividades econômicas que consequentemente alteram o uso do solo (Valle et al., 2016).

Uma das variáveis hídricas do ciclo hidrológico que existe grande potencial de mudança ao longo do tempo é a de vazões dos corpos d’água. As vazões de bacias hidrográficas podem apresentar tendências de aumento ou redução expressiva, provocando impactos generalizados sobre os demais recursos naturais e atividades antrópicas (Souza-Filho et al., 2015).

Além do conhecimento do ciclo hidrológico e da vazão de corpo d'água, o conhecimento do uso e ocupação do solo se torna necessário na tomada de decisões diante da realidade atual da ocupação desordenada e, devido principalmente ao descumprimento da atual legislação ambiental, os problemas existentes nas bacias hidrográficas fazem com que seja necessária a elaboração de diagnósticos, visando o correto uso/prognóstico de forma a melhorar o entendimento dos fatores naturais e artificiais que venham contribuir com a minimização dos problemas (Júnior \& Barbassa, 2012). Portanto, diante desse cenário, as técnicas de geoprocessamento se mostram eficientes para classificar uma área quanto ao uso e ocupação do solo, visto que são ferramentas que facilitam na observação das modificações das características naturais do ambiente, além de possibilitar o entendimento das possíveis causas e efeitos (Valadares, 2017).

Nessa perspectiva, a SRHI é uma Unidade de Planejamento da Região Hidrográfica Tocantins Araguaia, localizada no Estado do Pará, sendo o Rio Itacaiúnas o seu maior tributário devido aos municípios de Marabá e Parauapebas que são os mais importantes da Região Sudeste Paraense. A sub-região em estudo está situada em área de transição entre o bioma Cerrado e Amazônia e constam mudanças no uso da terra em razão da substituição de áreas florestais por atividades agropecuárias e mineração, que associados a outras atividades provocam desequilíbrio do regime hidrológico, que são fatores preponderantes para o aumento da temperatura, aumento das vazões anuais e degradação do solo (Souza-Filho et al., 2016). 
O estudo da SRHI é essencial, em virtude de a mesma apresentar múltiplas formas de uso dos seus recursos. Dessa forma, segundo IBGE (2018) atualmente a área de drenagem da sub-região é de aproximadamente $42.000 \mathrm{~km}^{2}$, abrigando 11 municípios que estão inseridos em toda a bacia, o que contribui para que o Produto Interno Bruto (PIB) seja de aproximadamente 270 bilhões de reais, através de atividades de pecuária, mineração, indústrias como, por exemplo, a siderúrgica, que fortalece o mercado e usufrui do recurso da bacia

Para que a visualização de uma bacia hidrográfica fique clara no contexto da percepção ambiental e consequentemente no manejo, torna-se interessante o emprego de mapas temáticos para representar os dados relacionados à área de interesse (Tucci, 2006). Portanto, o presente estudo objetiva analisar e caracterizar os efeitos da precipitação e do uso e cobertura do solo na vazão da Sub-Região Hidrográfica Itacaíunas (SRHI) nos períodos de 1988 a 2017.

\section{Metodologia}

A Sub-Região Hidrográfica Itacaíúnas (SRHI) objeto deste estudo (Figura 1), pertence à Região Hidrográfica Tocantins Araguaia referente a afluente do rio Tocantins e é uma divisão hidrográfica do Estado do Pará (ANA, 2008). A nível Federal, de acordo com ANA (2008) a bacia hidrográfica é considerada como Unidade de Planejamento Hídrico (UPH) por meio da delimitação socioeconômica e política. Nesse sentido, tanto a nível estadual quanto federal a SRHI apresenta a mesma delimitação para fins de planejamento hídrico.

A SRHI está localizada abaixo da linha do equador, visto que a área pertence à região do arco do desmatamento no bioma Amazônico, uma vez que possui uma área de drenagem de aproximadamente $41.300 \mathrm{~km}^{2}$. A área é delimitada por meio das coordenadas geográficas $05^{\circ} 10^{\prime}$ a $07^{\circ} 15^{\prime} \mathrm{S}$ latitude e $48^{\circ} 37^{\prime}$ a $51^{\circ} 25^{\prime}$ W longitude. O perímetro da unidade de planejamento em estudo intercede 11 municípios (Figura 1b) em que 6 deles possuem centros urbanos inseridos na área com um total de 700.000 habitantes e com produto interno bruto (PIB) de 270 bilhões de reais (Alvares et al., 2013).

Figura 1 - Localização da Sub-Região hidrográfica Itacaiúnas (SRHI).

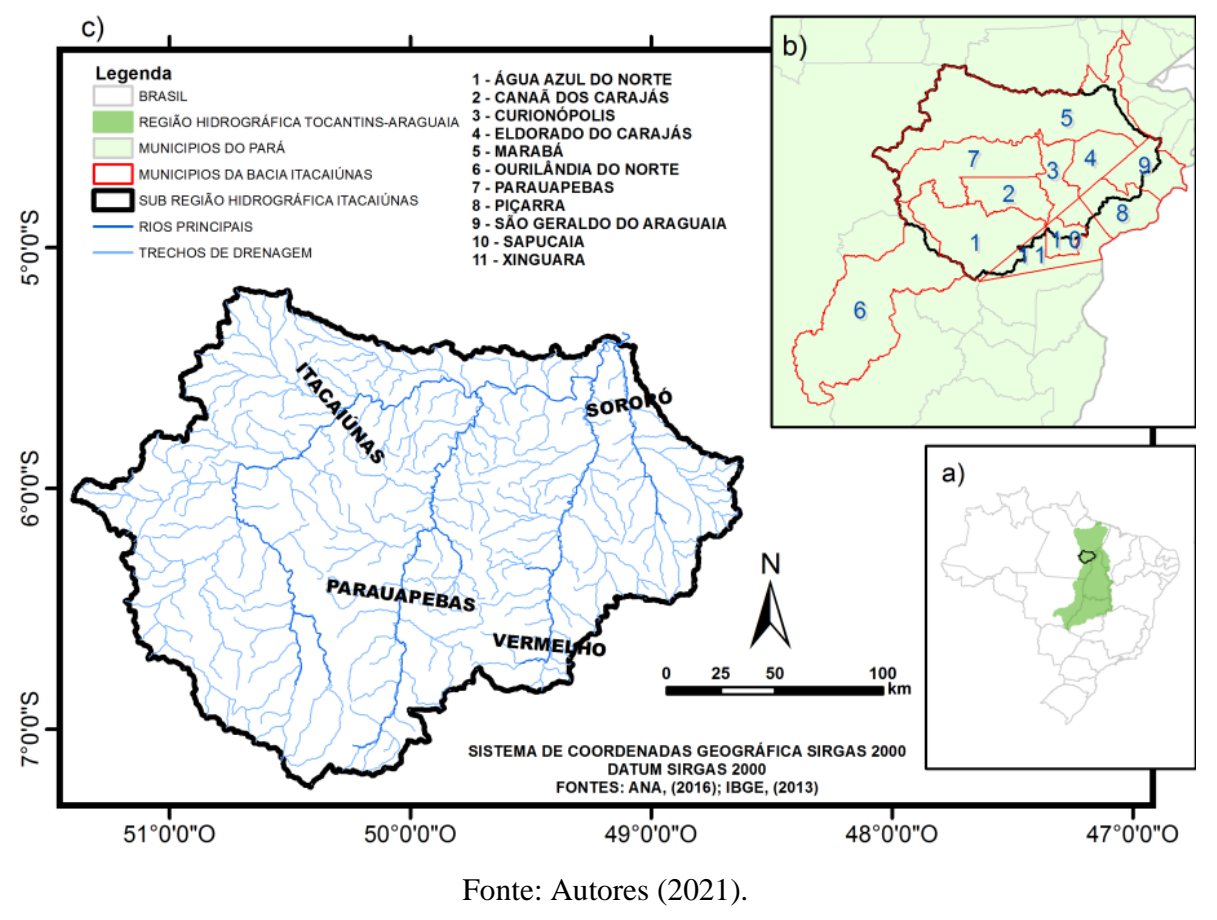


A metodologia adotada para a elaboração desse estudo foi aplicada, observacional e dedutiva. Quanto aos procedimentos técnicos efetuou-se o levantamento bibliográfico e documental para compor a fundamentação teórica através da avaliação de periódicos, com o intuito de construir novas hipóteses sobre a área de estudo. Nesse segmento, quanto à forma de abordagem, utilizou-se o método quali-quantitativo, posto que o estudo visa primeiramente atentar-se à qualidade em relação as características da área, para que posteriormente seja expressado de forma quantitativa os resultados obtidos com a aplicação da metodologia. Para isso, a pesquisa acontece de maneira retrospectiva quanto ao desenvolvimento no tempo, visto que o estudo visa abordar fatos passados e realinhar aos fatos ocorrentes na atualidade.

Para a obtenção dos dados de uso e ocupação do solo utilizou-se a plataforma do Projeto MapBiomas (MAPBIOMAS, 2019). Os arquivos disponibilizados são em rasters, uma vez que a plataforma já os classifica com valor de pixel entre 1 e 33 em que cada valor representa uma classe de uso do solo.

Na área de estudo, observou-se 10 tipos de usos do solo através do Projeto MapBiomas, são eles: Formação Florestal, Formação Savânica, Formação Campestre, Floresta Plantada, Outra Formação Natural não Florestal, Pecuária, Cultura Anual e Perene, Infraestrutura Urbana, Mineração e por último a classe Rio, Lagos e Oceanos.

No que diz respeito aos dados hidrológicos, foram obtidas séries históricas do banco de dados disponibilizados pela ANA (Agência Nacional de Águas) provenientes da plataforma HidroWeb (ANA, 2008). Por meio do software Hidro 1.5, houve o tratamento de dados pluviométricos e fluviométricos a partir da extração e exportação para uma planilha eletrônica, onde os dados obtidos foram entre os períodos de 1988 a 2017 para a correlação com mapeamento de uso do solo.

A associação entre vazões e uso do solo foi realizada por meio de análise qualitativa e quantitativa a partir dos dados da estação fluviométrica. As vazões utilizadas são vazão média anual (Qmed), vazão máxima anual (Qmax), vazão mínima anual (Qmin) (Lima, 2008).

\section{Resultados e Discussão}

O mapeamento do uso e ocupação do solo, como apresentado na Figura 2, expõe o período estudado de 1988 a 2017 , que foram analisados em intervalos de cinco anos. As classes que predominam na região correspondem ao Uso pecuário e a formação florestal, todavia as demais classes correspondem juntas menos de 5\% da área total da sub-bacia hidrográfica. 
Figura 2 - Uso e ocupação do solo da SRHI.

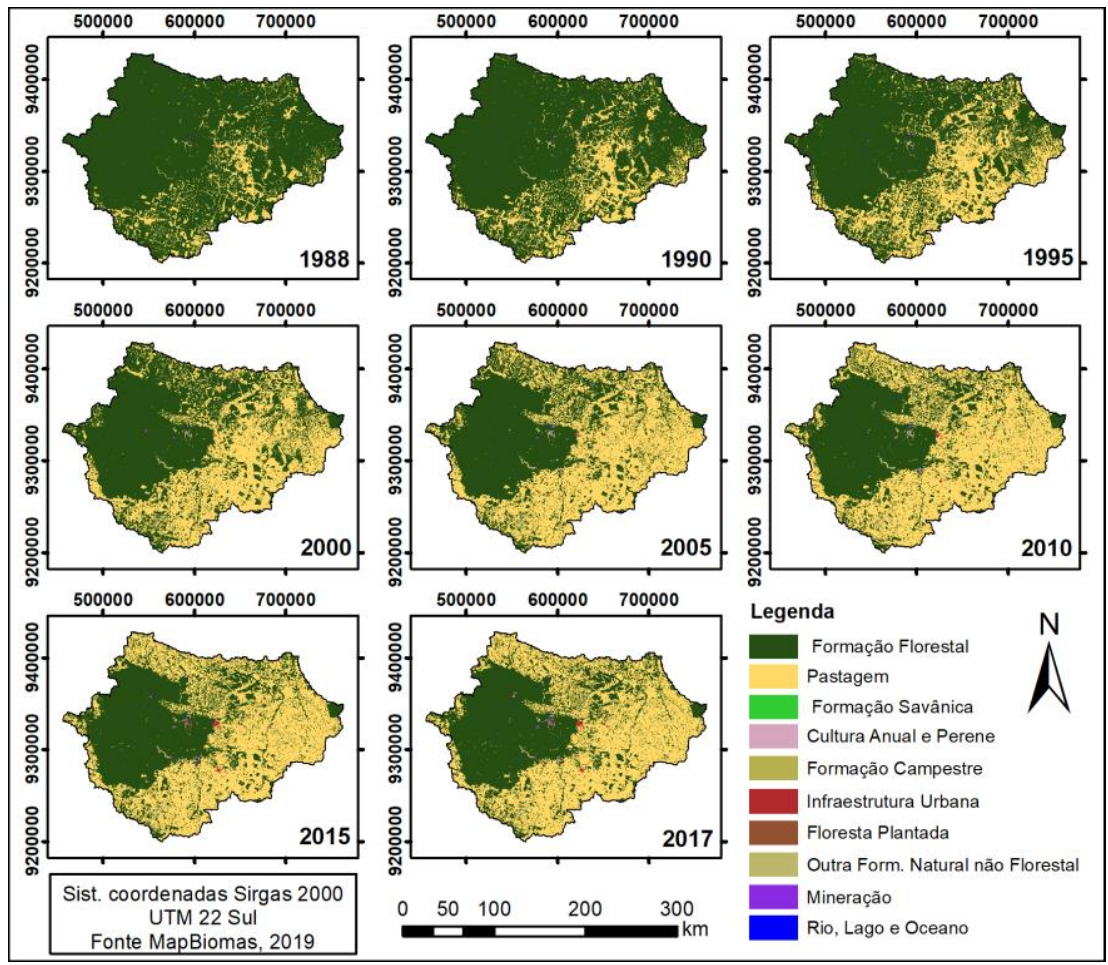

Fonte: Autores (2021).

Com isso, Godar et al., 2014, com o intuito de identificar a causa dos desmatamentos na Amazônia propôs que a principal causa do mesmo está relacionada com as vias de acessos aos interiores das florestas, intensificando as edificações e consequentemente a pecuária e a agricultura, assim como a exploração extrativista.

Em relação a precipitação e as vazões na região a Figura 3 proporciona visualizar o comportamento das variáveis no decorrer do tempo estudado. Nessa perspectiva, realizou-se as correlações de Spearman rho com a variável precipitação correlacionando com as vazões Qmin $(0,69)$, Qméd $(0,58)$ e Qmáx $(0,44)$. Com isso, observou-se que a correlação positiva não é muito expressiva onde a Qmin é a que mais se relaciona com a precipitação média da região.

Figura 3 - Vazões e precipitação entre 1988 a 2017 da SRHI.

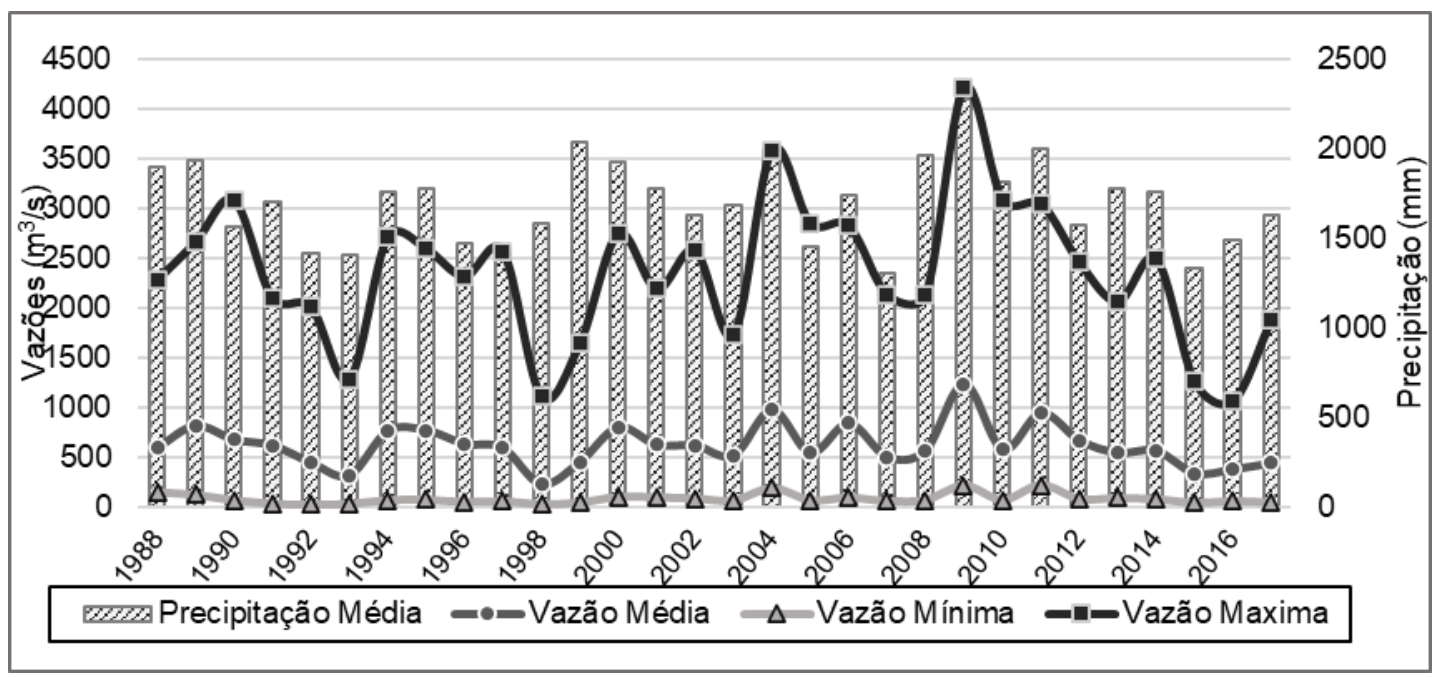

Fonte: Autores (2021). 
No que tange a análise temporal da vazão e o uso e ocupação do solo foi representado o comportamento das mesmas na Figura 4 em que é possível visualizar as vazões estudadas e as principais classes de uso e ocupação do solo encontradas na região. A correlação simples também foi feita em relação a variável Qméd com as classes formação florestal $(0,65)$ e a pastagem $(-0,65)$.

Figura 4 - Analise temporal da formação florestal, pastagem e vazões da SRHI.

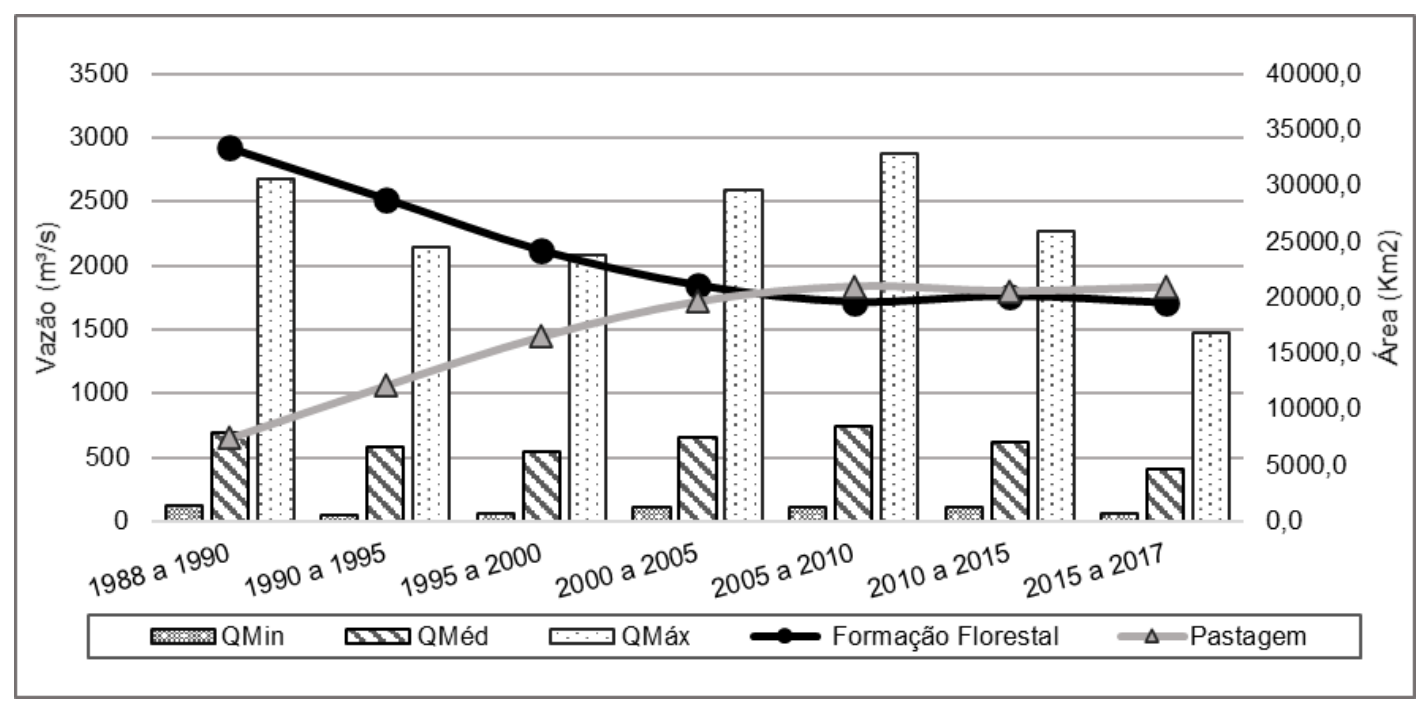

Fonte: Autores (2021).

Vanzela et al., (2010) ressalta que o uso e ocupação do solo intensivo influencia diretamente no escoamento e na infiltração da precipitação, com isso, o aumento da vazão média tende a aumentar conforme o uso do solo começa a ser mais expressivo. Porém, é possível visualizar que a relação vazão e uso e ocupação do solo não apresentou expressiva relação já que a classes de uso do solo apresentou uma dinâmica bem expressiva enquanto as vazões permaneceram quase constantes com dinâmicas não muito expressivas.

Através dessa relação, pode-se considerar que gramíneas de baixa produtividade, como pasto de pastagem natural, possuem menor área foliar e produzem menos detritos a vegetação original. Com uma área foliar inferior, a pastagem não intercepta tanta chuva quanto a floresta/cerrado, e uma fração maior atinge o chão. Com menos matéria orgânica no solo, a capacidade da superfície de detenção é diminuída e uma proporção maior da chuva escorre como escoamento superficial. (Costa et al., 2003).

Dessa forma, a relação vazão e floresta deveriam apresentar correlações proporcionais com valores de vazão, aumentando à medida que as áreas de pastagem aumentam. Segundo Pontes et al., (2019), a vazão de uma bacia hidrográfica além de ser afetada pelo uso do solo, outras variáveis também tem influência sobre a quantidade de água escoada, como por exemplo os tipos de solo e suas porosidades dessa maneira armazenando a água da chuva, e juntamente com a baixa precipitação.

\section{Conclusão}

A partir dos resultados obtidos pode-se concluir que o uso e ocupação do solo na Sub-Região Hidrográfica Itacaiúnas possuem pouca influência na dinâmica das vazões no trecho de contribuição estudado, porém as vazões estão estreitamente relacionadas com a precipitação da região.

É incontestável que a pesquisa não pode ser utilizada como referencial único de outros estudos voltados à temática de precipitação e uso e ocupação do solo nas vazões de bacias hidrográficas, visto que cada área possui distinções de outras, 
Research, Society and Development, v. 10, n. 15, e56101522520, 2021

sendo assim, torna-se necessário estudos mais elaborados e análises mais complexas de outros fatores e variáveis também importantes para o aprofundamento da pesquisa.

\section{Referências}

Adami, M. Gomes, A. R., Coutinho, A. C., Esquerdo, J. C. D. M., \&Venturieri, A. (2015). Dinâmica do uso e cobertura da terra no estado do Pará entre os anos de 2008 a 2012. João Pessoa-PB. Anais do XVII Simpósio Brasileiro de Sensoriamento Remoto - SBSR.

Agência Nacional de Águas - ANA (2008). Relatório Síntese: Plano Estratégico da Bacia Hidrográfica dos rios Tocantins e Araguaia. ANA. 204p.

Alvares, C. A., Stape, J. L., Sentelhas, P. C., Gonçalves, J. L. M., \& Gerd, S. (2013). Köppen's climate classification map for Brazil: Meteorologische Zeitschrift. 22, 711-728. http://dx.doi.org/10.1127/0941-2948/2013/0507.

Lei Federal Nº 9.433 de janeiro de 1997. (1997). Política Nacional de Recursos Hídricos. Diário Oficial da República Federativa do Brasil. Brasília, DF.

Costa, M. H., Botta, A., \& Cardille, J. A. (2003). Effects of large-scale changes in land cover on the discharge of the Tocantins River, Southeastern Amazonia: Journal of Hydrology: 283(1-4), 206-217.

Delazeri, L. M. (2016). Determinantes do Desmatamento nos Municípios do Arco Verde - Amazônia Legal: uma abordagem econométrica. Uberlandia-MG: Revista Economia - Ensaios. 30, 11-34.

ESRI. Environmental Systems Research Institute. (2010). Software ArcGIS desktop: version 10.6.1. Redlands: Environmental Systems Research Institute.

Godar, J., Gardner, T. A., Tizado, J., \& Pacheco, P. (2014). Actor-specific contributions to the deforestation slowdown in the Brazilian Amazon. USA: Proceedings of The National Academy of Sciences 11115591-15596.

Instituto Brasileiro de Geografia e Estatística - IBGE. (2018). Ministério do Meio Ambiente. Geociências: Downloads. https://downloads.ibge.gov.br/.

Júnior, Cláudio B., \& Barbasa, A. P. (2012). Geoprocessamento e recursos hídricos. São Carlos: Edufscar. p. 257.

Lima, W. P. (2008). Hidrologia Florestal Aplicada ao Manejo de Bacias Hidrográficas. (2a ed.), Universidade de São Paulo, p. 256.

MapBiomas. (2019). Coleção 4.0 da Série Anual de Mapas de Cobertura e Uso de Solo do Brasil. https://mapbiomas.org/download.

Pontes, P. R. M., Cavalcante, R. B. L., \& Sahoo, P. K. (2019). The role of protected and deforested areas in the hydrological processes of Itacaiúnas River Basin, eastern Amazonia: Journal of Environmental Management 235, 489-499.

Souza-Filho, P. W. M., Nascimento Júnior, W. R., Versiani, C. M., Silva Júnior, R. O., Guimarães, J. T. F., Dall’Agnol, R., \& Siqueira J. O. (2015). Changes in the land cover and land use of the Itacaiúnas river watershed, arc of deforestation, Carajás, southeastern amazon. Berlin: The International Archives of The Photogrammetry, Remote Sensing and Spatial Information Sciences, 1491-1496.

Souza-Filho, P. W. M., Souza, E. B., Silva Júnior, R. O., Nascimento Júnior, W. R., Mendonça, B. R. V., Guimarães, J. T. F., Dall'Agnol, R., \& Siqueira, O. S. (2016). Four decades of land-cover, land-use and hydroclimatology changes in the Itacaiúnas River watershed, southeastern Amazon. Journal of Environmental Management. 167, 175-184. http://dx.doi.org/10.1016/j.jenvman.2015.11.039.

Tucci, C. E. M.; \& Mendes, C. A. (2006). Avaliação Ambiental Integrada de Bacia Hidrográfica. Sqa, 302.

Valadares, A. A. (2017). Análise da dinâmica do uso e cobertura do solo sobre a vulnerabilidade ambiental em área do Distrito Federal. Dissertação Mestrado em tecnologia ambiental e recursos hídricos, Faculdade de Tecnologia, Universidade de Brasília, Distrito Federal.

Valle, I. C., Francelino, M. R., \& Pinheiro, H. S. K. (2016). Mapeamento da Fragilidade Ambiental na Bacia do Rio Aldeia Velha, RJ. Seropédica: Revista Floresta e Ambiente, 23, 201-215.

Vanzela, L. S., Hernandez, F. B. T., \& Franco, R. A. M. (2010). Influência do uso e ocupação do solo nos recursos hídricos do Córrego Três Barras, Marinópolis: Revista Brasileira de Engenharia Agrícola e Ambiental, 14, 55-64. http://dx.doi.org/10.1590/S1415-43662010000100008.

Vieira, I. C. G., Santos Junior, R. A. O., \& Toledo, P. M. (2014). Dinâmicas produtivas, transformações no uso da terra e sustentabilidade na Amazônia. In: Siffert Filho, Nelson Fontes et al. Um olhar territorial para o desenvolvimento: Amazônia. Banco Nacional de Desenvolvimento Econômico e Social, $370-395$. 